\title{
Managed Care Pharmacy Residencies and Fellowships and Other Programs
}

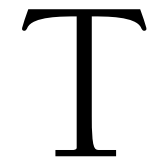
he following is a partial list of available managed care pharmacy residency and fellowship programs and other programs compiled as of August 2004. The residencies listed were submitted by AMCP members in response to AMCP's call for residency program listings. This is not a comprehensive list of all available programs. AMCP provides it solely as a

\section{ADVANCE PCS-A CAREMARK COMPANY}

\section{Managed Care}

Accredited: AMCP/ASHP

Length of Program: 12 months

Number of Positions: 4 ( 2 in Maryland, 1 in Texas, 1 in Arizona)

Affiliation:

University of Maryland, Texas Tech

University, University of Arizona

Application Deadline: 1/1/05

Starting Date: $\quad 7 / 1 / 05$

Estimated Stipend: $\quad \$ 33,300$

Onsite Interview: Yes

Educational/Special Requirements: PharmD or equivalent experience

Fringe Benefits: 2 weeks vacation, health insurance, free parking, professional meetings, other management, pharmaceutical industry

Special Features: Off-site rotations, university affiliation, ambulatory care clinic, disease management, industry experience

Contact Information:

Aaron Eaton

Center for Health Improvement

AdvancePCS-A Caremark Company

11350 McCormick Rd.

Executive Plaza II, Suite 1000

Hunt Valley, MD 21031

(410) $785-2182$

(410) 785-2140 (fax)

babette.edgar@advancepcs.com

\section{AMERICA SERVICE GROUP}

\section{Managed Care}

Accredited: Pending

Length of Program: 1-year or 2-year option

Number of Positions: 1

Affiliation: Various universities

Application Deadline: 2/28/05

Starting Date: $\quad 7 / 1 / 05$

Estimated Stipend: $\quad \$ 35,000$

Onsite Interview: Yes

Educational/Special Requirements: Graduate of accredited service to its readers. This list does not imply AMCP's endorsement of any particular program nor does AMCP guarantee the availability of any of the programs listed. AMCP does not assume responsibility for any errors that may appear in these listings. If you are aware of additional residency and fellowship programs not listed here, please contact AMCP at (800) TAP-AMCP.

school of pharmacy

Fringe Benefits: 3 weeks paid personal leave, paid sick leave, full medical and dental, tuition assistance toward specified degree programs

Special Features: America Service Group is a closed-model HMO that provides comprehensive at-risk health care. Secure Pharmacy Plus is the PBM.

Contact Information:

Peter Mikhail

Vice President Clinical Services

America Service Group/Secure Pharmacy Plus

416 Mary Lindsay Polk Dr., Suite 515

Franklin, TN 37067

(615) 771-1457

(615) $771-4557$ (fax)

peter.mikhail@securepp.com

\section{AMERICAN HEALTH CARE}

Clinical Therapeutics/Managed Care Pharmacy

Accredited: Being pursued

Length of Program: 12 months

Number of Positions: 1

Affiliation: None

Application Deadline: Open

Starting Date: $\quad 7 / 1 / 05$

Estimated Stipend: $\quad \$ 43,000$ and up

Onsite Interview: Yes

Educational/Special Requirements: PharmD with California pharmacist license or eligibility for license

Fringe Benefits: Medical, dental, vision, holidays and vacation, and attendance at national conference (s)

Special Features: Activities the resident will be involved in include (but are not limited to) P\&T Committee participation and presentations; formulary management and review; one-on-one physician correspondence on evidence-based medicine with current clinical studies reviewed and presented, focusing on actual patients that may benefit from this information; new drug review and its placement with available therapies; clinical participation and set-up; client summary report write-up and delivery; and participation in various education conference and clinical studies. 
Contact Information:

Nazly Westernoff

American Health Care

3001 Douglas Blvd., \#320

Roseville, CA 95661

(916) 773-7227

(916) 773-7210 (fax)

nazly@americanhealthcare.com

\section{AON CONSULTING}

\section{Pharmacy Benefits Consulting}

Accredited: $\quad$ No

Length of Program: 12 months

Number of Positions: 2

Affiliation: None

Application Deadline: 1/1/05

Starting Date: $\quad 7 / 1 / 05$

Estimated Stipend: $\quad \$ 36,000$

Onsite Interview: Yes

Educational/Special Requirements: PharmD

Fringe Benefits: 2 weeks paid vacation, paid holidays, medical/dental insurance, travel budget, professional meetings

Special Features: Aon Consulting is the third largest employee benefits consulting firm nationwide. This unique program provides residents with the opportunity to help employers and health plans better manage and control the prescription drug benefit for their employees or members. Residents will be exposed to all aspects of pharmacy benefit management consulting: plan design modeling, formulary analysis, clinical programming, disease management, audits, regulatory, trends and forecasting through proprietary actuarially based models. Residents will interact with benefit administrators, major PBMs, PPOs, HMOs, and disease management firms nationally. Currently, there are 2 resident positions available based in Chicago and New York City.

\section{Contact Information:}

Connie Perry, PharmD

Vice President

Aon Consulting

200 E. Randolph St., Suite 900

Chicago, IL 60601

(312) 381-4955

(312) 381-0239 (fax)

connie_f_perry@aoncons.com

\section{BLUE CROSS AND BLUE SHIELD OF ALABAMA}

\section{Managed Care}

Accredited:

AMCP/ASHP

Length of Program: 12 months

Number of Positions: 1

Affiliation: None

Application Deadline: 1/15/05

Starting Date: $\quad 7 / 1 / 05$ (flexible)

Estimated Stipend: $\quad \$ 32,000$
Onsite Interview: Yes

Educational/Special Requirements: PharmD or equivalent experience

Fringe Benefits: Paid vacation, personal holiday leave, health/dental insurance, no on-call responsibilities

Special Features: This program provides the resident with the opportunity to experience a true integrated medical and pharmacy system. The areas of focus will include pharmaceutical care, drug information, formulary management, clinical program management, disease state management, and outcome studies. The resident also will complete a research project suitable for publication. This program will incorporate communication and timemanagement skills.

Contact Information:

Jerry Wong, PharmD, MBA

Residency Director

BlueCross and BlueShield of Alabama

450 Riverchase Pkwy. East

Birmingham, AL 35244

(205) 220-6526

(205) 220-2939 (fax)

jwong@bcbsal.org

\section{BLUE SHIELD OF CALIFORNIA}

Managed Care Pharmacy Systems

Accredited: AMCP/ASHP

Length of Program: 12 months

Number of Positions: 1

Affiliation: UCSF

Application Deadline: 2/25/05

Starting Date: $\quad 7 / 1 / 05$

Estimated Stipend: $\quad \$ 46,000$

Onsite Interview: Yes

Educational/Special Requirements: PharmD degree from an accredited school of pharmacy, completion of a pharmacy practice residency or equivalent experience, 3 letters of recommendation, letter of intent, and on-site interview

Fringe Benefits: Health/dental/vision benefits; 20 days of paid time off, including professional leave (with travel allowances); 9 holidays; no on-call responsibilities

Special Features: This residency instills the philosophy that health care outcomes need to be considered from all relevant perspectives (patient, provider, and payer). Residents participate in the development of drug policy, clinical guidelines, pharmacy benefits, and population-based disease management; pharmaceutical contracting support/analysis; and quality improvement. This program teaches residents to conceptualize, integrate, and transform accumulated experiences and knowledge into improved drug therapy for managed care patients.

Contact Information:

Rosalie Patel

Clinical Pharmacist, Drug Information

Blue Shield of California Pharmacy Services 
50 Beale St., 22nd Floor

San Francisco, CA 94105

(415) 229-5693

(415) 229-6011 (fax)

rosalie.patel@blueshieldca.com

CAREMARK, INC.

\begin{tabular}{|c|c|}
\hline \multicolumn{2}{|c|}{ Managed Care Specialty-Analytics and Outcomes } \\
\hline Accredited: & No \\
\hline Length of Program: & 12 months \\
\hline Number of Positions: & 2 \\
\hline Affiliation: & $\begin{array}{l}\text { University of Illinois at Chicago; } \\
\text { Midwestern University-Chicago College } \\
\text { of Pharmacy }\end{array}$ \\
\hline Application Deadline: & $1 / 3 / 05$ \\
\hline Starting Date: & 7/1/05 \\
\hline Estimated Stipend: & $\$ 38,000$ \\
\hline Onsite Interview: & Yes \\
\hline
\end{tabular}

Educational/Special Requirements: PharmD with experiential or internship-based experience in the managed care/PBM industry

Fringe Benefits: Comprehensive medical, dental, and life insurance plan; 2-week paid vacation; holidays; employee stock purchase program; flexible spending program; travel budget

Special Features: Caremark is a leading pharmaceutical services company, providing comprehensive drug benefit services to approximately 24 million participants throughout the United States. Caremark's clients include corporate health plans, managed care organizations, insurance companies, unions, government agencies, and other funded benefit plans. The Analytics and Outcomes Residency will provide the resident with a unique opportunity to work on initiatives that foster proactive management of pharmaceutical and overall health care costs. It offers the ability to work with large data sets and perform various pharmaceutical cost analyses such as plan design modeling, formulary analysis, and clinical outcomes. As part of a core sales and account management team, the resident will have the opportunity to interact directly with clients, consultants, and various other benefit providers. While the focus is on analytics, the resident will be exposed to various areas in pharmacy benefit management such as clinical program development and implementation, operations, sales, account management, clinical sales support, marketing and communications, trade relations, pharmaceutical services, and therapeutic services.

Contact Information:

Anita Allemand

Manager, Client Analytic Servcies

Caremark, Inc.

2211 Sanders $R d$.

Northbrook, IL 60062

(847) 559-3923

(847) 559-5475 (fax)

anita.allemand@caremark.com
COVENTRY HEALTH CARE OF KANSAS, INC.

Pharmacy Benefits Management

Accredited: No

Length of Program: 12 months

Number of Positions: 1

Affiliation: $\quad$ University of Missouri, Kansas City (UMKC)

Application Deadline: 1/07/05

Starting Date: $\quad 7 / 1 / 05$

Estimated Stipend: $\quad \$ 30,000$

Onsite Interview: Yes

Educational/Special Requirements: PharmD degree from an ACPE-accredited college of pharmacy, licensed pharmacist in the United States or eligibility for licensure (successful candidate must be fully licensed at the start of residency), application, letter of intent, curriculum vitae, writing sample, official transcripts, 3 professional letters of recommendation, on-site interview with short formal presentation in Kansas City

Fringe Benefits: 2-weeks paid vacation, holidays, health insurance, paid travel and registration to AMCP Educational Conference, AMCP Annual Meeting, and ASHP Midyear Meeting

Special Features: Residency opportunities in all managed care core competencies: pharmacy, benefit management, utilization management, formulary management, clinical consultation service, drug information, new technologies assessment, provider network relations, Medicare plan, interventions, disease state management, health and wellness initiatives, quality assurance/ improvement activities, marketing and sales, contracting, health economics/outcomes, rotations with pharmaceutical industry

Contact Information:

Shawn Burke

Director of Pharmaceutical Services

Coventry Health Care of Kansas, Inc.

8320 Ward Pkwy.

Kansas City, MO 64114

(866) 795-3995

(866) 795-3992 (fax)

sburke@cvty.com

\section{DEPARTMENT OF VETERANS AFFAIRS MEDICAL CENTER}

Pharmacy Practice-Primary Care Emphasis

Accredited: ASHP

Length of Program: 12 months

Number of Positions: 3

Affiliation: None

Application Deadline: 1/14/05

Starting Date: $\quad 7 / 1 / 05$

Estimated Stipend: $\quad \$ 33,000, \$ 2,245$ FICA, $\$ 3,124$ other

benefits

Onsite Interview: Yes

Educational/Special Requirements: PharmD/equivalent experience Fringe Benefits: Vacation, paid holidays, sick days, and administrative time off for selected meetings

Special Features: ASHP-accredited pharmacy practice residency 
with large emphasis on direct patient care in a primary care setting. The pharmacy resident will work under a collaborative practice agreement with a medical team to facilitate achievement of therapeutic goals through evidence-based disease state management. In addition, the resident will have learning experiences in critical care, internal medicine, practice management, drug policy development, and education and teaching. Upon completion of this program, the pharmacy resident will have achieved a variety of advanced practice skills that will enable the graduate to feel confident to function effectively in multiple health care environments and roles.

\section{Contact Information:}

Jo-Ann Caudill

Residency Program Director

Department of Veterans Affairs Medical Center

3200 Vine St.

Cincinnati, $\mathrm{OH} 45220$

(513) 475-6322

(513) 475-6981 (fax)

Jo-Ann.Caudill@med.va.gov

\section{EHS}

\begin{tabular}{ll}
\hline Pharmacy Benefits Management \\
Accredited: & No \\
Length of Program: & 12 months \\
Number of Positions: & 1 \\
Affiliation: & University of Pittsburgh School of \\
& Pharmacy
\end{tabular}

Application Deadline: 1/12/05

Starting Date: $\quad 7 / 1 / 05$

Estimated Stipend: $\quad \$ 31,000$

Onsite Interview: Yes

Educational/Special Requirements: PharmD

Fringe Benefits: Comprehensive benefits package, no weekends/holidays, attend professional managed care meetings

Special Features: The University of Pittsburgh School of Pharmacy and EHS, one of the nation's largest pharmacy chainbased prescription management firms, offers an opportunity to practice in a dynamic PBM environment and gain a clinical and administrative perspective in managed pharmacy benefit plans for a wide variety of clients. Multifaceted experience will include DUR criteria development, clinical intervention activities, P\&T activities, clinical systems development, and new business development/ client services/marketing support.

\section{Contact Information:}

Teddi Gianangeli

Senior Clinical Management Specialist

EHS

620 Epsilon Dr.

Pittsburgh, PA 15238

(412) $967-2300$, ext. 5045

(412) 968-2676 (fax)

tgianang@ehs.com
GROUP HEALTH COOPERATIVE

\section{Managed Care Pharmacy Practice}

\section{Accredited: AMCP/ASHP}

Length of Program: 12 months

Number of Positions: 2

Affiliation: None

Application Deadline: 1/10/05

Starting Date: $\quad 7 / 1 / 05$

Estimated Stipend: $\quad \$ 40,000$

Onsite Interview: Yes

Educational/Special Requirements: PharmD or equivalent expe-

rience

Fringe Benefits: Full medical coverage ( $\$ 35 /$ month paid by the employee) and dental coverage (cost varies depending on plan chosen by employee) for the resident; 7 days of vacation; paid registration and some fees to attend professional meetings

Special Features: The residents are trained in the role of the pharmacist in the development and implementation of clinical practice guidelines, formulary development and management, as well as drug use policy development. In addition, residents are trained to function as leaders in implementing pharmaceutical care plans for specific patients in a managed care setting.

Contact Information:

Jim Carlson, PharmD

Director, Pharmacy Administration

Group Health Cooperative

12400 E. Marginal Way S

Seattle, WA 98168

(206) $901-4420$

(206) $901-4410$ (fax)

carlson.j@ghc.org

\section{HARVARD VANGUARD MEDICAL ASSOCIATES}

\section{Managed Care Pharmacy}

Accredited: ASHP

Length of Program: 12 months

Number of Positions: 1

Affiliation: Massachusetts College of Pharmacy and Health Sciences

Application Deadline: 1/7/05

Starting Date: $\quad 7 / 1 / 05$

Estimated Stipend: $\quad \$ 30,000$

Onsite Interview: Yes

Educational/Special Requirements: BS in Pharmacy or PharmD

Fringe Benefits: Comprehensive medical plan, 2 weeks paid vacation, professional travel allowance

Special Features: None

Contact Information:

William McCloskey

Massachusetts College of Pharmacy \& Health Sciences

179 Longwood Ave.

Boston, MA 02115 
(617) $732-2167$

(617) $732-2244$ (fax)

wmccloskey@mcp.edu

\section{HEALTH PARTNERS}

Managed Care Pharmacy

Accredited: No

Length of Program: 12 months

Number of Positions: 1

Affiliation: None

Application Deadline: 1/15/05

Starting Date: $\quad 7 / 1 / 05$

Estimated Stipend: Competitive

Onsite Interview: Yes

Educational/Special Requirements: PharmD

Fringe Benefits: Health insurance, vacation and holidays

Special Features: Travel/registration for 1 national meeting

Contact Information:

Vyvy Vo

Clinical Pharmacy Program Manager

HealthPartners

8100 34th Ave. South

P.O. Box 1309

Minneapolis, MN 55440

(952) 967-5133

(952) 883-5875 (fax)

vyvy.k.vo@healthpartners.com

\section{HENRY FORD HEALTH SYSTEM}

Managed Care Pharmacy

Accredited: AMCP/ASHP

Length of Program: 12 months

Number of Positions: 1

Affiliation: None

Application Deadline: 1/15/05

Starting Date: $\quad 7 / 1 / 05$

Estimated Stipend: $\quad \$ 30,000$

Onsite Interview: Yes

Educational/Special Requirements: PharmD; pharmacy practice residency desirable

Fringe Benefits: Health care, 2 weeks paid vacation, travel to 1 meeting

Special Features: The resident will design system enhancements and participate in ongoing utilization management, disease management, and compliance intervention programs. He/she will participate in formulary management and design clinical indications of effectiveness for guidelines. The resident will formulate and answer a research question, using scientific principles, with a strong emphasis on outcomes, pharmacoeconomics, and qualityof-life research. Experiences will include exposure to our HMO (Health Alliance Plan) and involvement with the Center for Clinical Effectiveness, Health System studies, Quality Improvement Center, and clinical pharmacy services. Direct patient care responsibilities in one of our ambulatory clinics will be ongoing throughout the year.

Contact Information:

Vanita Pindolia

VP, Pharmacy Care Management

Henry Ford Health System

30100 Telegraph Rd., Suite 200

Bingham Farms, MI 48025

(248) 723-0206

(248) 642-6094 (fax)

vpindoli@hapcorp.org

\section{HORIZON NJ HEALTH}

Managed Care Pharmacy

Accredited: No

Length of Program: 12 months

Number of Positions: 1

Affiliation: Horizon BC

Application Deadline: 2/1/05

Starting Date: $\quad 7 / 1 / 05$

Estimated Stipend: $\quad \$ 35,000$

Onsite Interview: Yes

Educational/Special Requirements: PharmD or equivalent experience; eligible for New Jersey state licensure

Fringe Benefits: Paid vacation, full medical/dental/retirement benefits

Special Features: Medicaid managed care HMO, unique focus on government programs, pharmacy case management, formulary and disease state management, development of clinical policies, outcomes research, assist with PharmD student oversight, ambulatory care experience, professional development courses, attendance to at least 1 national conference

Contact Information:

Samuel Currie, RPh

Director, Clinical Pharmacy Programs

Horizon NJ Health

210 Silvia St.

West Trenton, NJ 08628

(609) 538-0700

(609) 538-1698 (fax)

samuel_currie@horizonnjhealth.com

HUMANA, INC.

Managed Care Pharmacy

Accredited: $\quad$ AMCP/ASHP

Length of Program: 12 months

Number of Positions: 2

Affiliation: None

Application Deadline: 1/7/05

Starting Date: $\quad 7 / 1 / 05$

Estimated Stipend: $\quad \$ 32,000$

Onsite Interview: Yes

Educational/Special Requirements: PharmD preferred 
Fringe Benefits: Health, dental, 401K, 3 weeks vacation, relocation allowance

Special Features: None

Contact Information:

Teresa Watson-Heidari

Director, Outcomes Analysis

Humana, Inc.

500 W. Main St.

Louisville, KY 40202

(502) 580-3538

(502) 508-3538 (fax)

twatson-heidari@humana.com

IBA HEALTH PLANS/BLUE CARE NETWORK

\section{Managed Care Pharmacy}

Accredited: AMCP/ASHP

Length of Program: 12 months

Number of Positions: 1

Affiliation: $\quad$ Ferris State University/Michigan State University-Kalamazoo

Application Deadline: 1/15/05

Starting Date: $\quad 7 / 1 / 05$

Estimated Stipend: $\quad \$ 32,000$

Onsite Interview: Yes

Educational/Special Requirements: PharmD

Fringe Benefits: Health/dental, insurance, 2 weeks vacation, paid holidays

Special Features: Travel and registration reimbursement for 1 national conference, Great Lakes Residency Conference, and Pharmacy Residents conference; no weekend or evening shifts Contact Information:

Teresa Klepser, PharmD

Residency Director

MSU-KCMS/Ferris

1000 Oakland Dr.

Kalamazoo, MI 49008

(269) 337-6392

(269) 337-4474 (fax)

klepser@kcms.msu.edu

\section{INTERMOUNTAIN HEALTH CARE (IHC) HEALTH PLANS}

\section{Managed Care Pharmacy Practice}

Accredited: $\quad$ Anticipated July 2005

Length of Program: 1 year

Number of Positions: 1

Affiliation: None

Application Deadline: 1/10/05

Starting Date: $\quad$ 6/30/05

Estimated Stipend: $\quad \$ 40,000$

Onsite Interview: Required

Educational/Special Requirements: Candidates must have graduated from an ACPE-accredited pharmacy program with a doctor of pharmacy degree with a minimum GPA of 3.0 on a 4.0 scale; candidate must obtain Utah pharmacist licensure within the first 60 days of the program

Fringe Benefits: Health, dental, and life insurance; staff discounts; 10 days of vacation leave; and 10 holidays

Special Features: Travel benefits to either the ASHP Midyear Clinical Meeting or the AMCP Educational Meeting and the Western States Conference

Contact Information:

Douglas Burgoyne

Pharmacy Manager

IHC Health Plans

4646 W. Lake Park Blvd., Suite N3

Salt Lake City, UT 84120

(801) 442-5702

(801) 442-3006 (fax)

doug.burgoyne@ihc.com

\section{JANSSEN}

\section{Drug Information}

Accredited: No

Length of Program: 12 months

Number of Positions: 3

Affiliation: None

Application Deadline: 1/15/05

Starting Date: $\quad 7 / 1 / 05$

Estimated Stipend: n/a

Onsite Interview: Yes

Educational/Special Requirements: None

Fringe Benefits: None

Special Features: None

Contact Information:

Danielle Iobbi, PharmD

Manager, Medical Services

Janssen Pharmaceutica

1125 Trenton Harbourton Rd.

Titusville, NJ 08560

(609) 730-4493

(609) $730-3138$ (fax)

iobbi@janus.jnj.com

\section{KAISER PERMANENTE}

\section{Managed Care}

Accredited: $\quad$ No

Length of Program: 12 months

Number of Positions: 2

Affiliation: None

Application Deadline: January annually

Starting Date: July annually

Estimated Stipend: $\quad \$ 44,220$

Onsite Interview: Yes

Educational/Special Requirements: BS/PharmD. This is a specialty residency in managed care with emphasis on pharmacy practice management. 
Fringe Benefits: Medical benefits, 10 days sick/vacation leave, 5 holidays, $\$ 500$ for national meeting, plus expenses paid for Western States Residency meeting

Special Features: Emphasis in administration/practice management Contact Information:

Susan Downard, RPh

Area Pharmacy Manager

Kaiser Permanente

16601 E. Centretech Pkwy.

Aurora, CO 80011

(303) 326-6764

(303) 739-3574 (fax)

Susan.L.Downard@kp.org

KAISER PERMANENTE MEDICAL CARE PROGRAMINLAND EMPIRE SERVICE AREA

$\begin{array}{ll}\text { General Pharmacy Practice } \\ \text { Accredited: } & \text { ASHP } \\ \text { Length of Program: } & 12 \text { months } \\ \text { Number of Positions: } & 2 \\ \text { Affiliation: } & \text { None } \\ \text { Application Deadline: } & 1 / 15 / 05 \\ \text { Starting Date: } & 7 / 1 / 05 \\ \text { Estimated Stipend: } & \$ 35,000 \\ \text { Onsite Interview: } & \text { Yes }\end{array}$

Educational/Special Requirements: Graduate of accredited college of pharmacy and licensed or eligible for California licensure, pharmacy school transcript, 3 letters of recommendation, letter of intent, and curriculum vitae

Fringe Benefits: 2 weeks paid vacation; health benefits, including dental/optical; paid holidays; office space; reimbursement for offsite experiences

Special Features: Kaiser Permanente is the nation's largest nonprofit health plan serving more thant 8.4 million members in 9 states. The Inland Empire Service Area consists of 2 medical centers and 14 satellite medical offices for 600,000 members in the San Bernardino and Riverside counties of southern California.

Contact Information:

Patricia Gray

Clinical Operations Manager

Kaiser Permanente Inland Empire Service Area

9310 Sierra Ave.

Fontana, CA 92335

(909) 427-3838

(909) 427-3830 (fax)

patricia.l.gray@kp.org

KAISER PERMANENTE MEDICAL CARE PROGRAM — LOS ANGELES MEDICAL CENTER

Pharmacy Practice

Accredited: ASHP

Length of Program: 12 months

Number of Positions: 1
Affiliation: None

Application Deadline: 1/10/05

Starting Date: $\quad$ 7/1/05

Estimated Stipend: $\quad \$ 35,000$

Onsite Interview: Yes

Educational/Special Requirements: Graduate of accredited college of pharmacy and licensed or eligible for California licensure; good communication skills required. Resident will develop a project with targeted care outcomes and present at Annual Western States Conference.

Fringe Benefits: Medical, dental, optical insurance; holidays, vacation/sick leave

Special Features: The Kaiser Permanente Los Angeles Medical Center is the tertiary care center for Kaiser Permanente in Southern California and provides comprehensive inpatient, outpatient, and ambulatory care services to Kaiser Permanente members. This residency program provides development and training for recently graduated pharmacists, with an emphasis on pharmaceutical care and leadership to a diverse community. This program will allow residents to become familiar with pharmacy practice in an integrated health care program.

Contact Information:

Steve Litsey

Pharmacy Leader-Metro Service Area

Kaiser Permanente Pharmacy Operations Services

1515 N. Vermont Ave., Suite 237

Los Angeles, CA 90027

(323) 783-8306

(323) 783-7609 (fax)

toni.a.rodriguez@kp.org

KAISER PERMANENTE MEDICAL CARE PROGRAMWEST LOS ANGELES

Pharmacy Practice

Accredited: ASHP

Length of Program: 12 months

Number of Positions: 2

Affiliation: None

Application Deadline: 1/15/05

Starting Date: $\quad 7 / 1 / 05$

Estimated Stipend: $\quad \$ 35,000$

Onsite Interview: Yes

Educational/Special Requirements: Graduate of accredited college of pharmacy and licensed or eligible for California licensure. Good communication skills required. Resident will develop a project with targeted care outcomes and present at Annual Western States Conference.

Fringe Benefits: Medical, dental, optical insurance; holidays; vacation/sick leave

Special Features: Hospital and ambulatory care experiences in the nation's largest integrated care organization, preventative and disease state management in an integrated managed care setting. Flexible program molded to the resident's interests. 
Contact Information:

Michael Cinnamond, PharmD

Inpatient Pharmacy Director; Residency Program Director

Kaiser Permanente Medical Care Program at West Los Angeles

6041 Cadillac Ave., Suite B310

Los Angeles, CA 90034

(323) $857-2044$

(323) 857-2870 (fax)

michael.d.cinnamond@kp.org

KAISER PERMANENTE OF CALIFORNIA

\section{Medical Care/Drug Information}

Accredited: ASHP

Length of Program: 12 months

Number of Positions: 2

Affiliation: None

Application Deadline: After 2/05

Starting Date: $\quad 7 / 1 / 05$

Estimated Stipend: $\quad \$ 41,000$

Onsite Interview: Yes

Educational/Special Requirements: PharmD, pharmacy practice residency program or equivalent experience, excellent communication skills, eligible for California licensure, 3 letters of recommendation

Fringe Benefits: Medical, dental, optical insurance; 10 days time off; attendance at 1 pharmacy conference

Special Features: None

Contact Information:

Mirta Millares, PharmD

Kaiser Permanente

12254 Bellflower Blvd.

Downey, CA 90242

(562) 658-3630

(562) 572-3781 (fax)

mirta.millares@kp.org

\section{KAISER PERMANENTE OF COLORADO}

\section{Cardiology/Managed Care}

Accredited: No

Length of Program: 12 months

Number of Positions: 3

Affiliation: None

Application Deadline: 1/1/05

Starting Date: $\quad 7 / 1 / 05$

Estimated Stipend: $\quad \$ 41,220$

Onsite Interview: Yes

Educational/Special Requirements: PharmD required; prior pharmacy practice residency or equivalent preferred

Fringe Benefits: Health benefits, travel support to ASHP Midyear Meeting and Western States Residency Conference

Special Features: This cardiology specialty residency provides unique opportunities to acquire advanced knowledge and skills in ambulatory management of anticoagulation, cardiac risk reduc- tion, and heart failure in a managed care setting. Numerous educational opportunities exist. Experience in evaluating clinical and financial outcomes will be emphasized. Completion of a residency research project required.

Contact Information:

Anne Denham, PharmD

Clinical Pharmacy Specialist

Kaiser Permanente of Colorado

Clinical Pharmacy Cardiac Risk Service

16601 E. Centretech Pkwy.

Aurora, CO 80011

(303) 326-7663

(303) 326-7670 (fax)

anne.denham@kp.org

\section{KAISER PERMANENTE OF COLORADO}

\section{Mental Health/Managed Care}

Accredited: $\quad$ No

Length of Program: 12 months

Number of Positions: 1

Affiliations: None

Application Deadline: 1/1/05

Starting Date: $\quad 7 / 1 / 05$

Estimated Stipend: $\quad \$ 41,220$

Onsite Interview: Yes

Educational/Special Requirements: PharmD required; prior pharmacy practice residency or equivalent practical experience preferred

Fringe Benefits: Health benefits, travel support to ASHP MCM and Western States Residency Conference

Special Features: Experiences include inpatient as well as outpatient psychiatric pharmacy practice sites among full range of behavioral health conditions and neurology and chemical dependence. Evaluation of clinical outcomes is emphasized and completion of residency research is required.

Contact Information:

Daniel Dugan

Clinical Pharmacy Specialist in Mental Health

Kaiser Permanente of Colorado

16601 E, Centretech Pkwy.

Aurora, CO 80011

303-467-5776

303-467-5805 (fax)

daniel.j.dugan@kp.org

KAISER PERMANENTE OF COLORADO

Primary Care/Managed Care

Accredited: $\quad$ No

Length of Program: 12 months

Number of Positions: 3

Affiliation: None

Application Deadline: 1/1/05

Starting Date: $\quad 7 / 1 / 05$ 
Estimated Stipend: $\quad \$ 41,220$

Onsite Interview: Yes

Educational/Special Requirements: PharmD is required; prior pharmacy practice residency or equivalent experience is preferred Fringe Benefits: Health benefits, travel support to ASHP Midyear Meeting and Western States Residency Conference

Special Features: This Primary Care/Managed Care Specialty Residency provides the unique opportunity to acquire advanced knowledge and skills in ambulatory care pharmacotherapy in a managed care setting. Practice sites include primary care clinics, centralized services (anticoagulation, cardiac risk) and specialty services (diabetes, cardiology, infectious disease, mental health, nephrology). Residents participate in formulary management, pharmacoeconomic evaluations, and target drug programs. Numerous educational opportunities exist. Experience in evaluating clinical and financial outcomes will be emphasized. Completion of residency research project required.

Contact Information:

Rachana Patel, PharmD, BCPS, CDE

Clinical Pharmacy Specialist

Kaiser Permanente

1375 East 20th Ave.

Denver, CO 80205

(303) $764-4479$

(303) 861-3668 (fax)

rachana.j.patel@kp.org

\section{KAISER PERMANENTE OF GEORGIA}

\section{Managed Care Pharmacy Practice}

Accredited: AMCP/ASHP

Length of Program: 12 months

Number of Positions: 2

Affiliation: None

Application Deadline: 1/10/05

Starting Date: $\quad 7 / 1 / 05$

Estimated Stipend: $\quad \$ 34,000$

Onsite Interview: Yes

Educational/Special Requirements: PharmD preferred; eligible for Georgia licensure

Fringe Benefits: Medical/dental/vision, holidays, vacation/sick leave

Special Features: Ambulatory care, acute care, drug information, and administration

\section{Contact Information:}

Suzanne Booth, PharmD

Residency Program Coordinator

Kaiser Permanente

200 Crescent Centre Pkwy.

Tucker, GA 30083

(770) 496-3653

(770) 496-3487 (fax)

suzanne.booth@kp.org
MEDCO HEALTH SOLUTIONS, INC. Managed Care Pharmacy

Accredited: AMCP/ASHP

Length of Program: 12 months

Number of Positions: 2

Affiliation: None

Application Deadline: 1/15/05

Starting Date: $\quad 7 / 1 / 05$

Estimated Stipend: $\quad \$ 34,000$

Onsite Interview: Yes

Educational/Special Requirements: PharmD degree, eligible for New Jersey licensure; managed care rotation or experience preferred

Fringe Benefits: Complete medical coverage, paid holidays, and vacation

Special Features: Professional development trainings, travel, and attendance at educational meetings

Contact Information:

Becky Nagle

Senior Director, Clinical Practice and Education

Medco Health Solutions, Inc.

100 Parsons Pond Dr.

Mail Stop F2-3

Franklin Lakes, NJ 07417

(201) 269-6272

(210) 260-1035 (fax)

medcoRProg@medcohealth.com

\section{NOVARTIS PHARMACEUTICALS CORPORATION}

Health Economics \& Outcomes Research Fellowship

Accredited: No

Length of Program: 2 years

Number of Positions: 3

Affiliation: Duke University; Scott \& White Health

Plan/University of Texas at Austin

Application Deadline: 12/31/04

Starting Date: $\quad 7 / 1 / 05$

Estimated Stipend: $\quad \$ 35,000-\$ 44,000$

Onsite Interview: Yes

Educational/Special Requirements: Advanced degree in health services research, public health, health policy, pharmacy, economics, medicine, or other related areas, with some experience in outcomes research

Fringe Benefits: Medical insurance, vacation

Special Features: The fellows will gain familiarity with outcomes research principles/application and experience in designing research studies that examine economic, clinical, and humanistic outcomes. The first year is spent at an academic/managed care institution and the second year with Novartis's Health Economics $\&$ Outcomes Research Department.

Contact Information:

Feride Frech, RPh, MPH

Director, Health Economics \& Outcomes Research 
Novartis Pharmaceuticals Corporation

One Health Plaza

East Hanover, NJ 07936-1080

(862) 778-5094

(973) 781-3018 (fax)

feride.frech@pharma.novartis.com

OPTIMA HEALTH PLAN/SENTARA HEALTHCARE

Managed Care

Accredited: No

Length of Program: 12 months

Number of Positions: 1

Affiliation: None

Application Deadline: 2/15/05

Starting Date: $\quad 7 / 1 / 05$

Estimated Stipend: $\quad \$ 31,720$

Onsite Interview: Yes

Educational/Special Requirements: PharmD from an accredited school of pharmacy or equivalent experience, on-site interview, and eligible for Virginia licensure

Fringe Benefits: 2 weeks of paid vacation, uninterrupted stipend during minor illness, health insurance plan; travel assistance for continuing education events and other professional activities

Special Features: Resident will have exposure to an integrated health care system and system-wide pharmacy services, including a Drug Information Center. The resident will have the opportunity to precept students from Virginia Commonwealth University and Hampton University Schools of Pharmacy and work with medical residents from the Eastern Virginia Medical School.

Contact Information:

Elizabeth Brusig, PharmD

Clinical Pharmacist

Optima Health Plan

4417 Corporation Ln.

Virginia Beach, VA 23464

(757) $552-7519$

(757) 552-7516 (fax)

elbrusig@sentara.com

\section{OUTCOMES PHARMACEUTICAL HEALTH CARE}

\section{Medication Therapy Management Firm}

Accredited: $\quad$ ASHP accreditation pending

Length of Program: 1 year

Number of Positions: 1

Affiliation: University of lowa

Application Deadline: 12/31/04

Starting Date: $\quad 7 / 4 / 05$

Estimated Stipend: $\quad \$ 32,000$

Onsite Interview: Yes

Educational/Special Requirements: Candidates for residency must possess a doctor of pharmacy degree from a school of pharmacy accredited by the Accreditation Council for Pharmacy Education (ACPE) and be eligible for licensure in Iowa
Fringe Benefits: For additional information, contact the Outcomes office at (515) 237-0001 or info@getoutcomes.com. Special Features: Outcomes' Clinical Services Residency is a 12-month managed care systems residency program. It offers a unique learning experience on how pharmacists can impact the quality and rising cost of health care by providing patient-oriented, care-based services. Residency activities include account management, marketing and communication, pharmacoeconomic data collection and analysis, claims assessment, development and implementation of disease management program, development and administration of research projects, training of pharmacists and support staff, and serving as a preceptor to PharmD clerkship students.

Contact Information:

Patty Kumbera

Chief Operations Officer

Outcomes Pharmaceutical Health Care

601 E. Locust, Suite 200

Des Moines, IA 50309

(515) 237-0001

(515) 237-0002 (fax)

pkumbera@getoutcomes.com

\section{PHARMACEUTICAL CARE NETWORK}

\section{Managed Care Pharmacy Practice}

Accredited: No

Length of Program: 12 months

Number of Positions: 1

Affiliation: None

Application Deadline: 1/15/05

Starting Date: $\quad$ 7/1/05

Estimated Stipend: $\quad \$ 37,500$

Onsite Interview: Yes

Educational/Special Requirements: Graduate of accredited college of pharmacy and licensed or eligible for California licensure

Fringe Benefits: Paid vacation and sick leave, health/dental/vision benefits, and educational support to attend professional meetings Special Features: This residency will provide training in formulary management, provider drug therapy education, drug benefit design, outcomes analysis, and prior authorization. The resident will participate in drug utilization review and P\&T committee presentations, and interface with pharmacists from a variety of professional fields. The resident will learn to use PCN's MedIntelligence software to identify drug therapy problems and make appropriate interventions. This program includes rotations in direct patient care, drug information, and an opportunity to rotate through the California Pharmacists Association.

Contact Information:

Philip Parsatoon

Vice President, Professional Services

9343 Tech Center Dr., Suite 200

Sacramento, CA 95826-2563

(916) 361-4450 
(916) 414-4650 (fax)

resident@pharmcarenet.com

PHARMACY MANAGEMENT CONSULTANTS/ OKLAHOMA UNIVERSITY

\section{Managed Care Pharmacy}

Accredited:

ASHP—Managed Care Pharmacy

Systems

Length of Program: 12 months

Number of Positions: 1

Affiliation: Oklahoma University College of Pharmacy

Application Deadline: 2/1/05

Starting Date: $\quad 7 / 1 / 05$

Estimated Stipend: $\quad \$ 35,000$

Onsite Interview: Preferred

Educational/Special Requirements: Pharmacy degree

Fringe Benefits: 10 days vacation, health and dental coverage, faculty appointment at the level of clinical instructor, sponsorship to at least 1 professional meeting

Special Features: Evaluate health plan formularies, develop clinical guidelines, evaluate retrospective and prospective DUR criteria, develop educational intervention programs, serve as preceptor for pharmacy students; experience working with dual Medicare/Medicaid eligibles

Contact Information:

Elgene Jacobs, $P h D$

Pharmacy Management Consultants

1122 NE 13th

ORI W-4403

Oklahoma City, OK 73117

(405) 271-9039, ext. 47354

(405) 271-1647 (fax)

elgene-jacobs@ouhsc.edu

\section{PRESCRIPTION SOLUTIONS}

Managed Care

Accredited: ASHP

Length of Program: 12 months

Number of Positions: 1 or 2

Affiliation: None

Application Deadline: 1/7/05

Starting Date: $\quad 7 / 5 / 05$

Estimated Stipend: $\quad \$ 40,000$

Onsite Interview: Yes

Educational/Special Requirements: PharmD, licensed to practice in California

Fringe Benefits: 2 weeks paid vacation, paid holidays, sick days, travel reimbursement

Special Features: Drug therapy management, developing clinical guidelines for appropriate drug use, formulary management, legal and regulatory affairs, professional meetings and seminars, (required to attend the ASHP Midyear Clinical Meeting, CSHP Seminar,
AMCP Educational Conference, and Western State Conference)

Contact Information:

Alex Gilderman

Director, Residency Program

C/O Prescription Solutions

3515 Harbor Blvd.

Costa Mesa, CA 92626

(714) 825-6768

(714) 825-3742 (fax)

alex.gilderman@rxsol.com

\section{RUTGERS UNIVERSITY/}

HORIZON BLUE CROSS BLUE SHIELD OF NEW JERSEY

Managed Care Organization

Accredited: $\quad$ No

Length of Program: 12 months

Number of Positions: 2

Affiliation: Rutgers University, the State University

Application Deadline: 1/15/05 of New Jersey

Starting Date: $\quad 7 / 1 / 05$

Estimated Stipend: $\quad \$ 30,000$

Onsite Interview: Yes

Educational/Special Requirements: PharmD; eligible for New Jersey state license

Fringe Benefits: Full medical coverage, dental and retirement benefits

Special Features: No weekend or staffing requirements; teaching and preceptoring, if desired; graduate courses offered at Rutgers University; industry and PBM perspectives of managed care

Contact Information:

Saira A. Jan, MS, PharmD

Associate Professor, Rutgers University

Associate Director, Pharmacy Management at Horizon BCBSNJ

Rutgers University/Horizon Blue Cross Blue Shield of NJ

Three Penn Plaza East PP-13Q

Newark, NJ 07105

(973) 466-4575

(973) 466-6266 (fax)

saira_jan@horizon-bcbsnj.com

\section{UNIVERSITY OF BUFFALO}

Ambulatory Care

Accredited: Pending

Length of Program: 1 year

Number of Positions: 2

Affiliation: University of Buffalo

Application Deadline: 10/1/04

Starting Date: $\quad$ 1/01/2005

Estimated Stipend: TBD

Onsite Interview: Required

Educational/Special Requirements: PharmD or equivalent experience required 
Fringe Benefits: health, dental, vision, paid vacation, conference travel funds

Special Features: Unique experience designed to further refine skills in pharmaceutical care in addition to developing skills in program development and personnel and resource management. Resident is involved in coordination of clinical activities in a highvolume, lobby-based pharmacy and a health-clinic-based ambulatory care pharmacy. The resident will participate in medication histories, adherence counseling, and education programs. Development and implementation of disease management initiatives, patient education, medical informatics, and supervision of PharmD students are also significant aspects. This residency will allow ample latitude for resident to explore interests and further develop skills as a practitioner. The resident will also be appointed as a clinical instructor at University of Buffalo, School of Pharmacy and Pharmaceutical Sciences.

Contact Information:

Gene Morse, PharmD

University of Buffalo

School of Pharmacy and Pharmaceutical Sciences

311 Hochstetter Hall

Buffalo, NY 14260

(716) $645-2828$

(716) 645-2886 (fax)

emorse@buffalo.edu

\section{UNIVERSITY OF HOUSTON/KELSEY-SEYBOLD CLINIC}

\section{Pharmacy Practice With an Emphasis on Managed Care}

Accredited: ASHP/AMCP

Length of Program: 1 year

Number of Positions: 2

Affiliation: University of Houston

Application Deadline: 1/2/05

Starting Date: $\quad 7 / 1 / 05$

Estimated Stipend: $\$ 30,000$

Onsite Interview: Yes

Educational/Special Requirements: PharmD or equivalent experience

Fringe Benefits: State of Texas benefits, 10 days of vacation, support to attend AMCP, ASHP, TSHP, and either Alcalde or Mid-West Residency Conference

Special Features: Ambulatory care-focused, administrative and clinical responsibilities, collaborations with other $\mathrm{UH}$ residents

Contact Information:

Sarah Lake-Wallace, PharmD

Kelsey-Seybold Clinic

Pharmacy Administration

8900 Lakes at $610 \mathrm{Dr}$.

Houston, TX 77054

(713) $442-6248$

(713) $442-5253$ (fax)

slwallace@kelsey-seybold.com
UNIVERSITY OF ILLINOIS AT CHICAGO AND WALGREENS HEALTH INITIATIVES

\section{Fellowship-Outcomes Research}

Accredited: No

Length of Program: 2 years

Number of Positions: 1

Affiliation: $\quad$ University of Illinois and Walgreens

Health Initiatives

Application Deadline: 2/1/05

Starting Date: $\quad 7 / 1 / 05$

Estimated Stipend: $\quad \$ 36,000$

Educational/Special Requirements: PharmD or MD (or equivalent) and completion of a pharmacy practice or managed care residency

Special Features: This is a 2-year fellowship jointly offered by Walgreens Health Initiatives and the Center for Pharmacoeconomic Research at the University of Illinois at Chicago. The aim of the program is to train clinical pharmacists to conduct research in drug therapy outcomes and pharmacoeconomics in the managed care setting. Knowledge and experience will be gained in the use of research tools to evaluate economic, humanistic, and clinical outcomes of drug therapy. Presentation and publication of research findings in peer-reviewed venues is expected. The fellowship is designed to facilitate career opportunities in managed care, health provider organizations, consulting, academia, or the pharmaceutical industry.

Contact Information:

Glen Schumock, PharmD, MBA

University of Illinois at Chicago

Center for Pharmacoeconomic Research

833 S. Wood St. (MC 886)

Chicago, IL 60612

(312) 996-7961

schumock@uic.edu

\section{UNIVERSITY OF MARYLAND SCHOOL OF PHARMACY/CARE FIRST BLUE CROSS BLUE SHIELD}

\section{Managed Care Pharmacy}

Accredited: $\quad$ No

Length of Program: 12 months

Number of Positions: 1

Affiliation: University of Maryland

Application Deadline: 1/15/05

Starting Date: $\quad$ 7/1/05

Estimated Stipend: $\quad \$ 31,500$

Onsite Interview: Yes

Educational/Special Requirements: Graduate degree in pharmacy Fringe Benefits: Health insurance, parking, support for national meeting attendance and poster presentation

Special Features: Appointment as a clinical instructor at the University of Maryland School of Pharmacy, ambulatory care clinics at HMO; office with computer/references at managed care organization 
Contact Information:

Catherine Cooke, PharmD

Clinical Assistant, Adjunct Professor

University of Maryland School of Pharmacy

5106 Bonnie Branch Rd.

Ellicott City, MD 21043

(410) 480-5012

(410) 480-5296 (fax)

catherine.cooke@pfizer.com

\section{WALGREENS HEALTH INITIATIVES}

PBM

Accredited:

AMCP/ASHP

Length of Program: 12 months

Number of Positions: 2

Affiliation: $\quad$ University of Illinois; Midwestern University-Chicago College of Pharmacy

Application Deadline: 1/7/05

Starting Date: $\quad 7 / 5 / 05$

Estimated Stipend: $\quad \$ 36,000$

Onsite Interview: Yes

Educational/Special Requirements: PharmD

Fringe Benefits: Medical plan, 2-week vacation, holidays, travel expense budget

Special Features: This managed care pharmacy residency program is designed to allow the residents to work within the various departments of a pharmacy benefits management firm including, but not limited to, care management, drug use policy, PBM operations, clinical sales, and specialty pharmacy. The residents will gain practical experience and develop skills related to disease management, health outcomes, medication management strategies, formulary management, drug utilization review, drug information, and other clinical services. Additionally, residents will have the opportunity to gain exposure to the pharmaceutical industry, be involved in professional organizations, and precept pharmacy students.

Contact Information:

Susie Min

Manager, Clinical Education and Shared Faculty

Walgreens Health Initiatives

1417 Lake Cook Rd., MS\# L457

Deerfield, IL 60015

(847) $964-6740$

(847) 374-2669

susie.min@walgreens.com

\section{WELL POINT PHARMACY MANAGEMENT}

\section{Managed Care Pharmacy}

Accredited: $\quad$ Being pursued

Length of Program: 12 months

Number of Positions: 1

Affiliation: BlueCross of California, Precision Rx, University of Southern California

Application Deadline: 1/15/05 $\begin{array}{ll}\text { Starting Date: } & 7 / 1 / 05 \\ \text { Estimated Stipend: } & \text { TBD } \\ \text { Onsite Interview: } & \text { Yes }\end{array}$

Educational/Special Requirements: PharmD, or equivalent experience; eligible for California licensure

Fringe Benefits: Health insurance, paid vacation and holidays, attendance at the Western States Conference

Special Features: PBM, health plan, mail order, and direct patient care experience. This program is designed to provide the resident with an overall managed care experience. The resident will rotate through several areas within the PBM, including clinical intervention analysis and policy, clinical account management, pharmaceutical contracting and industry relations, clinical product development, drug information, therapy management, clinical intervention and prior authorization centers, and mail order. The experience will also include experiential sites at a California-based health plan and clinics at the University of Southern California.

Contact Information:

William Waugh, PharmD

Director, Clinical Affairs

WellPoint Pharmacy Management

8407 Fallbrook Ave. AF-7

West Hills, CA 91304

(818) 313-5077

(818) 313-5110 (fax)

william.waugh@wellpoint.com

As of press time, these residency listings had not yet been updated for 2005. These are the listings as they appeared for 2003/2004. Please contact the preceptors for more information.

CLINICAL PHARMACOLOGY SERVICES, INC.

Ambulatory Care/Clinical Research

Accredited: No

Length of Program: 12 months

Number of Positions: 1

Affiliation: None

Application Deadline: 2/1/04

Starting Date: $\quad 6 / 1 / 04$

Estimated Stipend: $\quad \$ 34,000$

Onsite Interview: Yes

Educational/Special Requirements: None

Fringe Benefits: Sponsorship to professional meeting and Southwestern Residency Conference

Special Features: None

Contact Information:

Daniel Buffington, Director

Clinical Pharmacology Services, Inc.

6825 E. Fowler Ave.

Tampa, FL 33617

(813) 983-1500

(813) 983-1501 (fax)

danbuffington@cpshealth.com 
KAISER FOUNDATION HEALTH PLAN OF THE MID-ATLANTIC STATES, INC.

\section{Managed Care Pharmacy}

Accredited: AMCP/ASHP

Length of Program: 12 months

Number of Positions: 2

Affiliation: None

Application Deadline: 1/9/04

Starting Date: $\quad 7 / 1 / 04$

Estimated Stipend: $\quad \$ 34,000$

Onsite Interview: Yes

Educational/Special Requirements: PharmD preferred; pharma-

cy licensure eligibility in $\mathrm{DC}, \mathrm{MD}$, or $\mathrm{VA}$

Fringe Benefits: Medical benefits; selected holidays; sick, vacation, and education leave

Special Features: In addition to helping develop new and innovative programs, the resident will participate on the Pharmacy $\&$ Therapeutics Committee, teach patient education classes, provide pharmacy staff continuing education, assist in educating pharmacy students, and complete residency projects and presentations.

Contact Information:

Katrin Fulginiti

Director, Managed Care Pharmacy Practice Residency Program

Kaiser Permanente-Mid Atlantic

12201 Plum Orchard Dr.

Silver Spring, MD 20904

(301) $572-3330$

(301) 572-3399 (fax)

katrin.fulginiti@kp.org

\section{KAISER PERMANENTE MEDICAL CARE PROGRAM-}

\section{CALIFORNIA}

\begin{tabular}{ll}
\hline Pharmacy Practice and Drug Information Practice \\
Accredited: & ASHP \\
Length of Program: & 12 months \\
Number of Positions: & 26 \\
Affiliation: & None \\
Application Deadline: & $1 / 15 / 04$ \\
Starting Date: & $7 / 1 / 04$ \\
Estimated Stipend: & $\$ 35,000$ \\
Onsite Interview: & Yes
\end{tabular}

Educational/Special Requirements: Graduate of accredited college of pharmacy and licensed or eligible for California licensure. Good communication skills required. Resident will develop a project with targeted care outcomes and present at Annual Western States Conference.

Fringe Benefits: Medical, dental, optical insurance; holidays; vacation/sick leave

Special Features: Hospital and ambulatory care experiences in the nation's largest health maintenance organization, preventative and disease state management in an integrated managed care setting Contact Information:
Elaine Watanabe, PharmD

Pharmacy Services Manager_-Recruitment

Kaiser Permanente Pharmacy Operations Services, California Division 9521 Dalen St.

Downey, CA 90242

(714) 796-4809

(714) $796-4826$ (fax)

Elaine.G.Watanabe@kp.org

KAISER PERMANENTE MEDICAL CARE PROGRAMTRI-CENTRAL

Pharmacy Practice

Accredited: ASHP

Length of Program: 1 year

Number of Positions: 2

Affiliation: None

Application Deadline: 1/15/04

Starting Date: $\quad 7 / 1 / 04$

Estimated Stipend: $\quad \$ 35,000$

Onsite Interview: Yes

Educational/Special Requirements: Recent graduate of an accredited school of pharmacy and eligible for California licensure, college transcript(s), 3 letters of recommendation, curriculum vitae

Fringe Benefits: 2 weeks paid vacation; 6 paid holidays; sick leave; health benefits, including dental/optical (also dependents); uniforms; office space; and reimbursement for off-site experiences Special Features: The resident will become familiar with the various aspects of pharmacy practice in a large health maintenance organization. The residency includes experiences in acute and ambulatory care practice, drug information/drug-use policy development, and practice management. Experiences in drug information, asthma management, anticoagulation, hyperlipidemia, home health, and pharmacoeconomics are important segments of this residency. Time is allowed for the resident to pursue an area of particular interest. Residents participate on the P\&T Committee, teach patient-education classes, and serve as a preceptor for pharmacy students and pharmacy interns during the residency year. Contact Information:

Robert Endo

Clinical Operations Manager

Kaiser Permanente Medical Care Program, Pharmacy Operations

1011 Baldwin Park Blvd.

Baldwin Park, CA 91706

(626) 851-5307

(626) 851-5813 (fax)

robert.n.endo@kp.org

SCOTT \& WHITE

Pharmacy Practice Managed Care

Accredited: No

Length of Program: 1 year

Number of Positions: 1 
Affiliation: University of Texas at Austin

Application Deadline: 2/15/04

Starting Date: $\quad 7 / 1 / 04$

Estimated Stipend: $\quad \$ 30,000$

Onsite Interview: Yes

Educational/Special Requirements: PharmD; eligible for Texas

state license

Fringe Benefits: Full medical and dental coverage

Special Features: Minimal weekend staffing requirements; teaching and preceptoring, if desired; industry and PBM perspectives of managed care; marketing and sales exposure

Contact Information:

John Jackimiec

Director of Pharmacy Managed Care

Scott \& White

2601 Thornton Ln., Suite A

Temple, TX 76502

(254) $742-3144$

(254) 742-3109 (fax)

jjackimiec@swmail.sw.org

\section{SCRIP PHARMACY SOLUTIONS}

\section{Managed Care Pharmacy}

Accredited: No

Length of Program: 12 months

Number of Positions: 1

Affiliation: University of Rhode Island

Application Deadline: 1/31/03

Starting Date: $\quad 7 / 1 / 03$

Estimated Stipend: $\quad \$ 30,000+$

Onsite Interview: Yes

Educational/Special Requirements: None

Fringe Benefits: 10 days paid vacation, 9 paid holidays, health benefits, and professional travel allowance

Special Features: This 12-month residency program is designed to provide a firm knowledge base of managed care from the pharmacy benefit perspective and allows residents to excel in clinical services, formulary management and research, participation in formulary development and medication management, provision of clinical services including drug information, clinical program development, pharmaceoeconomic assessment, as well as the strengthening of written and verbal communication skills.

Contact Information:

Ingrid $\mathrm{Ma}$

Scrip Pharmacy Solutions

33 North Rd.

Wakefield, RI 02879

(877) 842-5097, ext. 6726

(401) 783-3520 (fax)

ima@scripsolutions.com

\section{UNITED DRUGS}

\section{Managed Care}

Accredited: $\quad$ No

Length of Program: 1 year

Number of Positions: 1

Affiliation: AMCP

Application Deadline: 6/15/04

Starting Date: $\quad$ 9/1/04

Estimated Stipend: $\quad \$ 33,000$

Onsite Interview: Yes

Educational/Special Requirements: Completion of degree from an accredited pharmacy school, PharmD preferred; ability to work independently

Fringe Benefits: Understanding of managed care from the perspective of a PBM. Product development opportunities. Ability to live in Arizona for a year!

Special Features: Work hours negotiable

Contact Information:

Jean Brown

Director of Clinical Services

United Drugs

7227 North 16th St., Suite 160

Phoenix, AZ 85020-5256

(602) 678-1179, ext. 229

(602) 678-0772 (fax)

brownj@uniteddrugs.com

UTMB CORRECTIONAL MANAGED CARE

Pharmacy Practice with an Emphasis in Managed Care

Accredited: ASHP

Length of Program: 12 months

Number of Positions: 1

Affiliation: UTMB

Application Deadline: 3/1/04

Starting Date: $\quad$ 6/1/04

Estimated Stipend:

Onsite Interview: Yes

Educational/Special Requirements: Pharmacy degree from accredited college of pharmacy, Texas pharmacy license

Fringe Benefits: Off major holidays, no weekends, no on-call, no dispensing

Special Features: Automation, televideo clinics, ambulatory care Contact Information:

Stephanie Zepeda

Asst. Director of Pharmacy

UTMB Correctional Managed Care

Estelle Unit-Pharmacy Bldg.

264 FM 3478

Huntsville, TX 77320

(936) 291-4200, ext. 3709

(936) 295-7012 (fax)

sdzepeda@utmb.edu 